\title{
Enhancing Micro-EDM using Ultrasonic Vibration and Approaches for Machining of Nonconducting Ceramics
}

\author{
Andreas Schubert1,2 - Henning Zeidler1,* - Matthias Hackert-Oschätzchen ${ }^{1}$ - Jörg Schneider ${ }^{2}$ - Martin Hahn1 \\ ${ }^{1}$ Chemnitz University of Technology, Chair Micromanufacturing Technology, Germany \\ 2Fraunhofer Institute for Machine Tools and Forming Technology IWU, Germany
}

\begin{abstract}
Micro EDM (Electro Discharge Machining) is a known nonconventional process for the machining of hard to cut materials. Due to its ablating nature based on melting and evaporation through heat induced by electrical discharges, it can function independently of the hardness, toughness or brittleness of the workpiece. Because of these benefits, EDM is widely used in tool- and mould making; micro-EDM, with its much lower discharge energies, has been successfully applied to micromachining of high-accuracy parts. The precision manufacturing of high aspect ratio micro geometries such as deep micro bores relies on stable process conditions in the discharge gap. Its minimisation - a precondition for minimal feature size and higher accuracy - limits the effectiveness of conventional flushing techniques, leading to a higher fraction of unwanted discharge states (open and short circuit), lower process speed, and geometrical errors. New hybrid technology approaches, such as ultrasonic or low frequency superposition, significantly raise the process stability and speed.

Another restriction on the use of EDM, the exclusive machinability of electrically conductive materials, is overcome by the application of the assisting electrode method that enables a micro-ED-machining of nonconductive zirconium oxide ceramics.

This paper presents the current status of investigation into the micro-EDM process with ultrasonic vibration assistance - directly applied to the workpiece and indirectly applied high-intensity ultrasonic to the dielectric - in metallic materials as well as in the machining of electrically nonconductive ceramic materials. Using ultrasonically aided micro-EDM, the process speed can be raised by up to $40 \%$, enabling bores of less than $90 \mu \mathrm{m}$ in diameter with aspect ratios $>40$ for metallic materials. The modified setup using the assisting electrode principle allows for machining of an aspect ratio $>5$ for nonconductive ceramic materials, leading to new possibilities for the design and manufacture of complex, high-accuracy micro parts in high-performance engineering materials.
\end{abstract}

Keywords: electro discharge machining, micro machining, ultrasonic, ceramics

\section{O INTRODUCTION}

\subsection{Micro-EDM}

Electro discharge machining (EDM) is a known and widely used nonconventional machining process for hard to cut materials because of its ability to function independently of the hardness, brittleness or toughness of the workpiece. Using low energies, it has been successfully applied in micro- and precision machining, for example, in the mould making industry.

Higher accuracies in smaller and more complex structures demand an extremely stable process and therefore enhanced flushing techniques.

Additionally, more and more high-tech materials such as ceramics are being used for micro parts; this presents an opportunity to apply $\mu \mathrm{EDM}$ in that emerging material area.

\subsection{Process Principle}

The electro discharge machining process is based on ablation of material through melting and evaporation. Fig. 1 shows the process principle.

The electrical discharges take place between the tool electrode and the workpiece in a dielectric medium that separates the two. A voltage is applied to both electrodes and, when the breakdown voltage of the medium is reached, a plasma channel allowing for a current flow is established and a discharge takes place. At the base of the plasma channel the temperature can reach $T \geq 10,000{ }^{\circ} \mathrm{K}$, melting and evaporating the electrode material.

When the energy input is stopped the discharge ends, leading to a collapse of the plasma channel and the surrounding gas bubble. The reflow of the dielectric medium flushes liquid material away and cools the electrode surface. Repeating the process, a voltage is attached to the electrodes again and the setup is prepared for the next discharge.

Naturally, the discharge will take place where the breakdown barrier is lowest, that is when the distance between the electrodes is the smallest - in an ideal dielectric - or, in a real dielectric liquid, when the conductivity of the gap between the electrodes is the highest, e.g. when particles or gas bubbles reduce the breakdown voltage of the medium.

By constant repetition of the process, the tool electrode surface is reassembled in the workpiece and, by feeding the tool, a transfer of the geometry takes place. Because of the process nature, the surface is an assembly of single discharges and shows a crater-like topology. The geometrical accuracy and the surface roughness depend on the size and shape of these craters and therefore on the volume that is ablated 


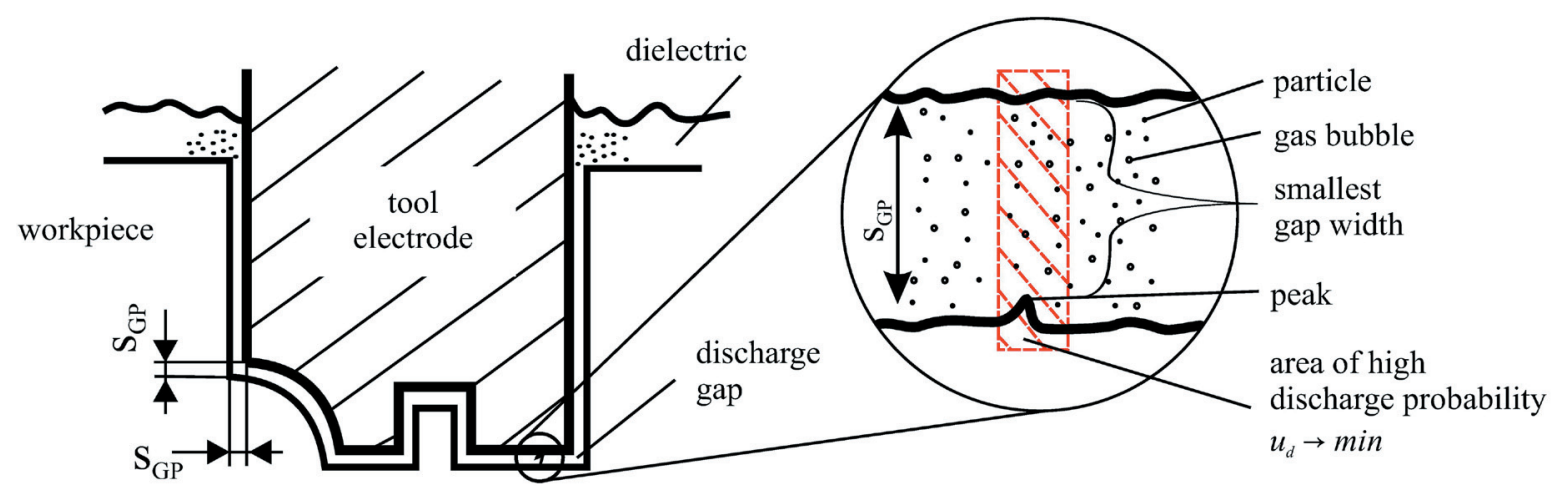

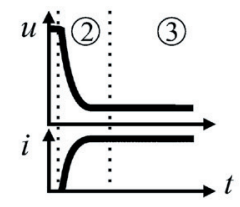

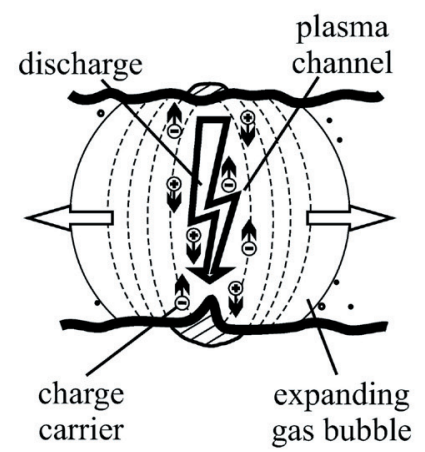

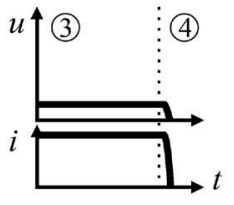

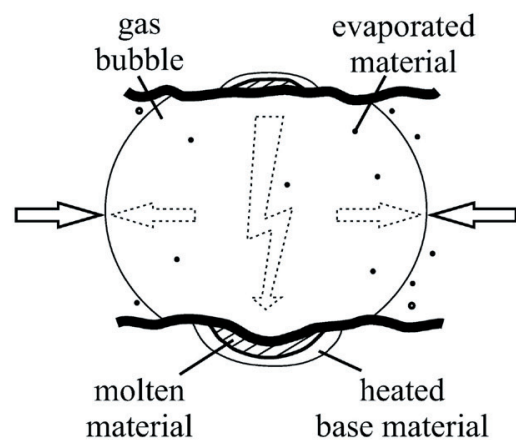

Fig. 1. EDM process principle
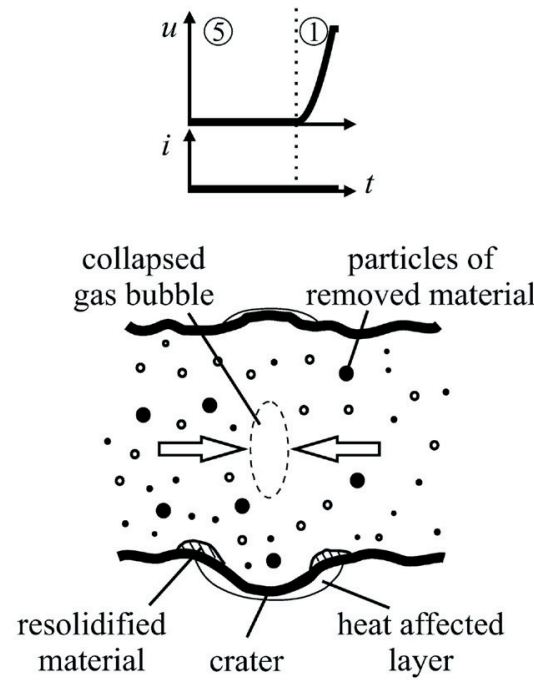

layer with each discharge. A minimisation of discharge energy is the key to precision and optimal surface characteristics. Consequently, the discharge gap must be minimised, too.

In $\mu \mathrm{EDM}$, the discharges have a typical duration of $t_{e} \approx 100 \mathrm{~ns}$ and transfer energy of $W_{e} \approx 10 \mu \mathrm{J}$. The resulting crater width depends on the workpiece material properties, but a diameter of $d_{C} \approx 5 \mu \mathrm{m}$ and depth of $\leq 1 \mu \mathrm{m}$ can usually be obtained. The resulting surface roughness can be as low as $R_{z} \leq 1 \mu \mathrm{m}$.

A major benefit of the electro discharge machining process, due to its electro-thermal nature of ablation, is independency of material hardness and brittleness. The noncontact nature of the process results in a nearly force-free machining, allowing the usage of soft, easy to machine electrode materials even when shaping very hard workpieces.

This also enables the machining of fragile or thin workpieces. Additionally, there is no limitation to the angle between the tool and workpiece, so round or irregularly shaped surfaces can be used.

For all those reasons, EDM has been widely used in the generation of micro parts and geometries such as spinnerets (Fig. 2) [1].

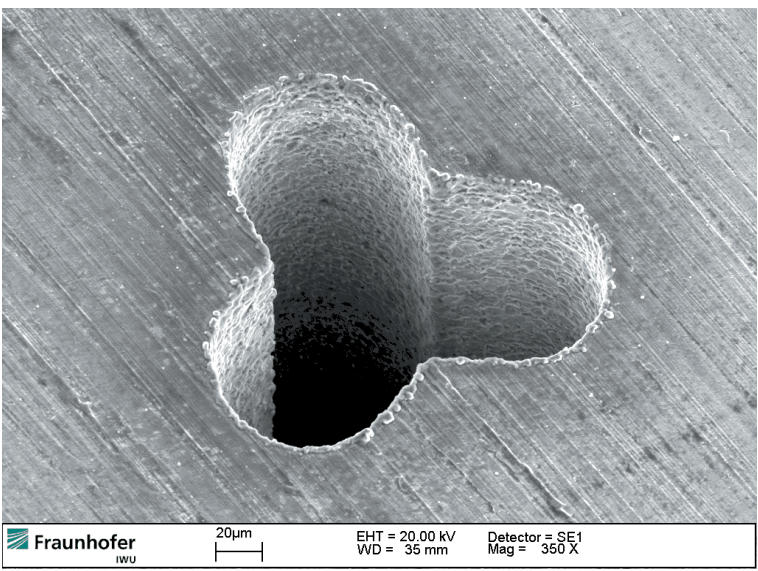

Fig. 2. SEM image of non-circular micro bore $\emptyset 80 \mu \mathrm{m}$ in hardened steel, manufactured using vibration-assisted $\mu E D M$ 


\subsection{Limiting Parameters}

An ideal EDM process delivers identical shape and energy for each discharge. In reality, this is not possible. The estimated efficiency ratio for $\mu \mathrm{EDM}$ is between 30 and $50 \%$ [2], meaning that the rest of the discharges are not optimal. Short circuits, open circuits or long arc discharges do not only impact effective machining, but also have negative effects on electrode wear, form distortion, and process speed.

An optimal discharge - in reality - only occurs if the environmental parameters are optimal at the time the discharge takes place. Therefore it is of great importance to deliver, if not optimal, at least constant environmental parameters to be able to find a suitable generator setup.

One way to achieve this is the regulation of the discharge gap width. Only at an optimum value, influenced by the properties of the medium filling the gap, can an efficient discharge take place.
The discharge gap width is commonly regulated based on the mean gap voltage $\left(u_{e}\right)$ that delivers different levels for the normal, short or open circuit state. Depending on this and a set target value, the electrode is fed or withdrawn (feed $s$ ) (Fig. 3).

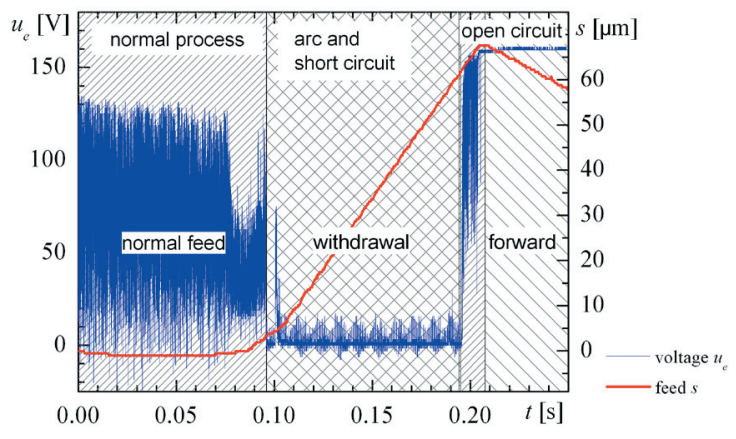

Fig. 3. Electrode feed as a reaction to gap voltage

Novel approaches at Fraunhofer IWU and TU Chemnitz aim to develop a current-based predictive

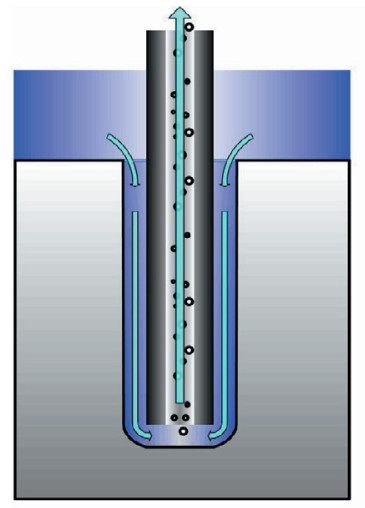

sucking pump flushing

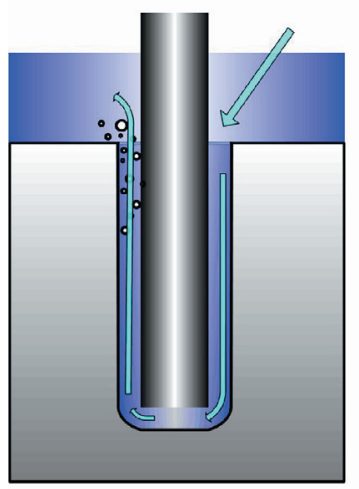

external jet flushing

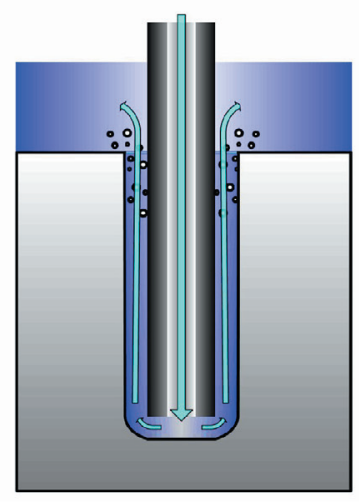

forced pump flushing

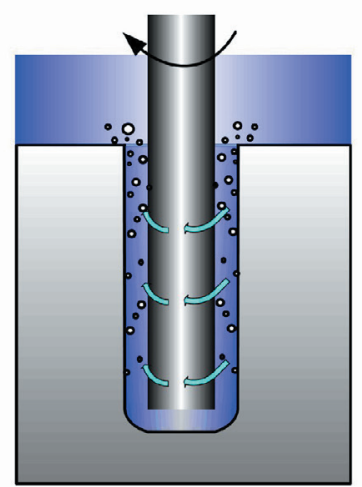

rotational flushing

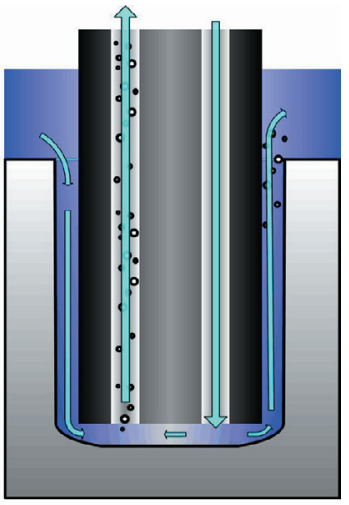

combined flushing

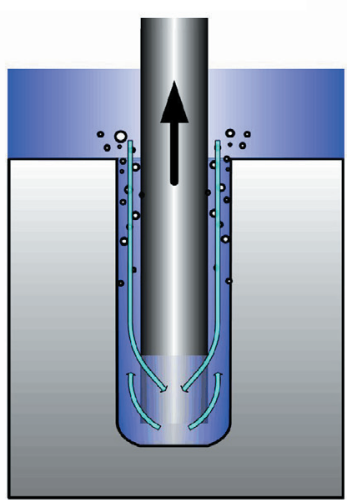

flushing by lifting dielectric liquid $\because \because \because 0$ ablated particles

Fig. 4. Flushing options in EDM 
process control to reduce the reaction delay and increase the efficiency ratio of $\mu \mathrm{EDM}$. However, since the discharge duration is in the range of $100 \mathrm{~ns}$, new algorithms and fast, microprocessor-based systems have to be implemented.

Of course, the more stable the gap conditions are, the less short-circuiting and time-consuming intervention of the gap width regulation occurs. An optimal gap flushing is therefore crucial.

High-aspect ratio micro bores with tool diameters of less than $100 \mu \mathrm{m}$, however, present the most difficult flushing conditions. In $\mu \mathrm{EDM}$, many conventional strategies of gap flushing (Fig. 4), such as high-pressure flushing or flushing through bores in the tool electrode, are, because of the small size and fragility of the tool, not available. The rotational flushing loses efficiency due to the small tool diameter and the resulting low circumferential speed.

Furthermore, as mentioned before, to achieve maximum precision, the discharge gap has to be minimised. Currently, in $\mu \mathrm{EDM}$, gap widths of wGP $\leq$ $10 \mu \mathrm{m}$ are common.

The combination of these factors leads to large difficulties in efficient flushing and new approaches have to be taken to ensure stable process conditions. One option is the direct vibration of the workpiece, which can be considered a high frequency version of the flushing by lifting principle.

\section{VIBRATION SUPERPOSITION}

\subsection{Effects on the EDM Process}

The effect of direct vibration superposition on the $\mu \mathrm{EDM}$ process was found to be very beneficial. The periodic relative movement between tool and workpiece causes a flow of the dielectric and an agitation of the debris particles in the dielectric medium. With this, the settling of debris on the bore ground and the agglomeration of particles are reduced and the state of the gap is equalised.

An improvement in terms of flushing and homogenisation of the dielectric liquid leads to a faster and more stable process with better form accuracy, allowing for higher aspect ratios and more complex structures. Since the vibration frequency directly influences the flow speed in the dielectric, ultrasonic and high-frequency vibrations are especially beneficial. However, since resonant systems are required for these frequencies, they present some difficulties in the implementation if they are to be applied directly to the workpiece.

\subsection{Low Frequency Vibration}

Compared to ultrasonic vibration, low-frequency vibration can be easily realised, since there is no need for resonant systems. Tests were conducted to characterise the effect of a LF-vibration on the machining of bore holes.

While the acceleration of process speed is in the region of $10 \%$ compared to conventional machining, a larger contribution can be seen in stabilising difficult geometries and reducing geometrical errors and process instabilities, which can be observed by a reduction of the machining time variation on the order of $>50 \%$ (Fig. 5, Section (2)).

However, the specific machine tool setup has to be taken into consideration when choosing the vibration frequency in order to avoid a negative interaction with the gap width regulation. Section (1) in Fig. 5 shows this phenomenon.

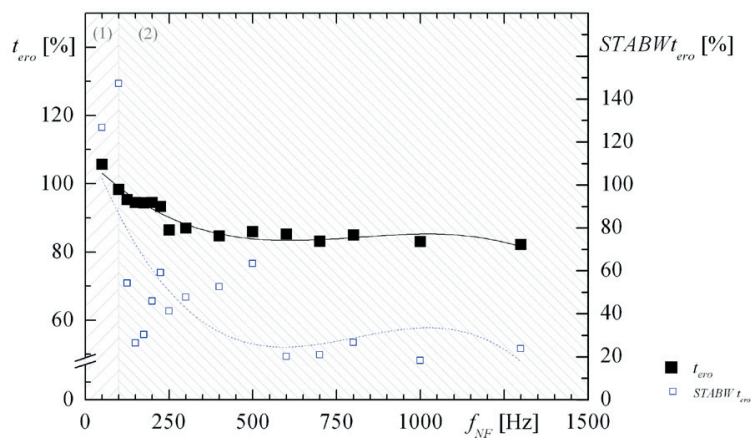

Fig. 5. Low-frequency vibration aided $\mu E D M$; effects on machining time $t_{\text {ero }}$ and its deviation

\subsection{Ultrasonic - Direct}

Direct ultrasonic vibration of the tool or workpiece (Fig. 6) is regarded as the optimal strategy for improved flushing and stabilised $\mu$ ED-machining of high aspect ratio structures. The high frequency in the range from 20 to $60 \mathrm{kHz}$ with amplitudes of 2 to 10 $\mu \mathrm{m}$ peak-peak significantly influences the state of the frontal discharge gap and therefore the process itself.

Two major effects can be distinguished: 1) the influence of the vibration on the dielectric and 2) the immediate influence to the working gap width through periodic feed-retraction-movements of the vibrated part.

The first effect can be described by a very high velocity movement of the dielectric. Its speed can reach more than $0.5 \mathrm{~m} / \mathrm{s}$ with accelerations of over 60 $\mathrm{km} / \mathrm{s}^{2}$, efficiently moving and stirring the dielectric. An agglomeration of particles is reduced, leading to a 
more uniform gap condition and therefore increasing the amount of efficient discharges.

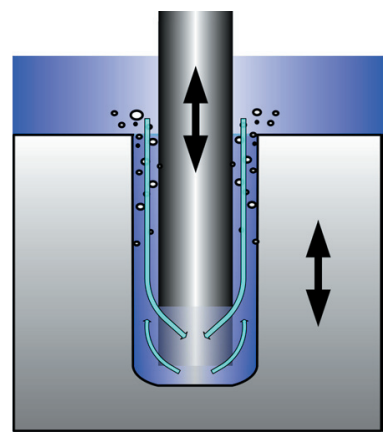

Fig. 6. Direct vibration of tool or workpiece in $\mu E D M$

The second effect relates to the periodic change in the gap width through vibration. There, a retracting movement ends longer arc discharges that cause geometric deviation and process instabilities [3].

As a result, the process speed is significantly enhanced and, additionally, more complex structures can be reproducibly machined. Fig. 7 shows the tool feed $s_{z}$ for the machining of very deep micro bores with an optimised conventional process and two direct ultrasonically assisted processes. The used tool electrode diameter is $60 \mu \mathrm{m}$ (a solid tungsten carbide rod, machined by centerless grinding).

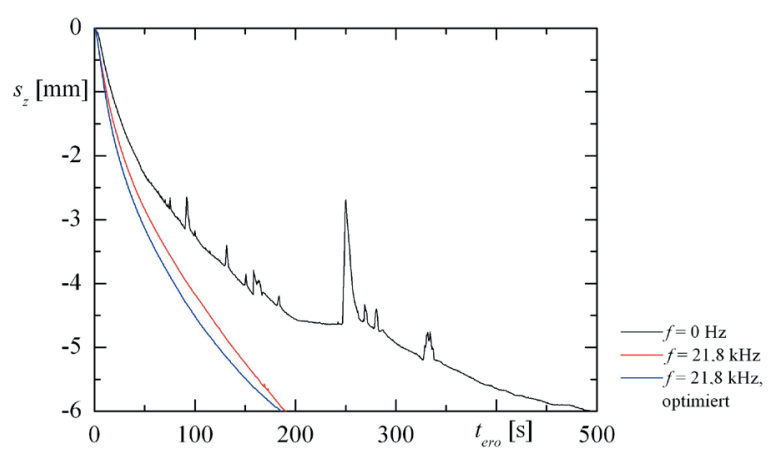

Fig. 7. Tool feed sz during deep bore $\mu$ EDM (tool $\varnothing 60 \mu \mathrm{m}$ )

It can be seen that with increasing depth the conventional process $(f=0 \mathrm{~Hz})$ not only slows down, but also becomes unstable, which leads to retracting movements of the tool electrode caused by long short circuit periods. For the direct ultrasonically assisted processes $(f=21.8 \mathrm{kHz})$, no large retracting movements are observed.

This allows for the machining of high aspect ratio structures such as micro bore arrays (bore diameter 85 $\mu \mathrm{m}$, depth $1000 \mu \mathrm{m}$, grid width $110 \mu \mathrm{m}$, steel, Fig. 8) or complex external structures such as the cone array shown in Fig. 9. The cone bottom radius is $75 \mu \mathrm{m}$, the top radius $8 \mu \mathrm{m}$, and its height $800 \mu \mathrm{m}$. The distance between the two cones (centre to centre) is $250 \mu \mathrm{m}$.

In deep boring experiments, micro bores of diameter $100 \mu \mathrm{m}$ and depths of $5000 \mu \mathrm{m}$ could be successfully machined in steel.

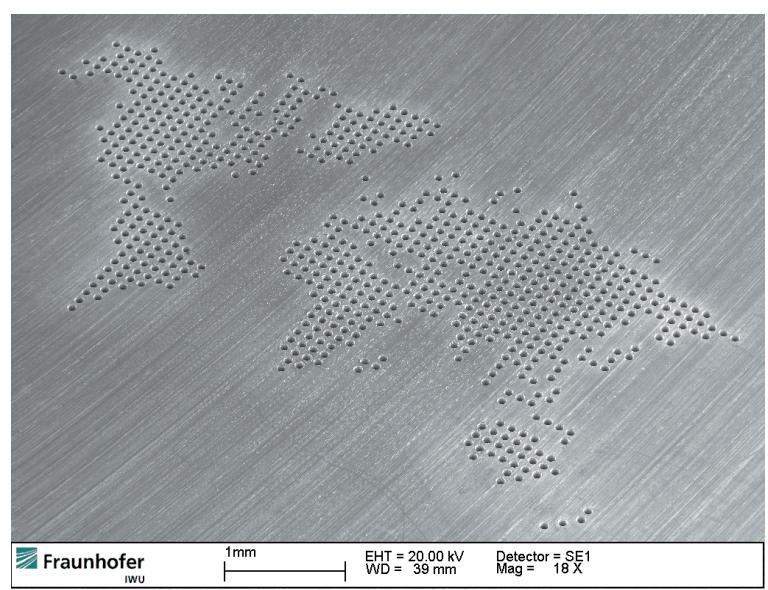

Fig. 8. Array of 751 micro bores, diameter $85 \mu$ m, depth 1000 $\mu \mathrm{m}$, grid width $110 \mu \mathrm{m}$, steel

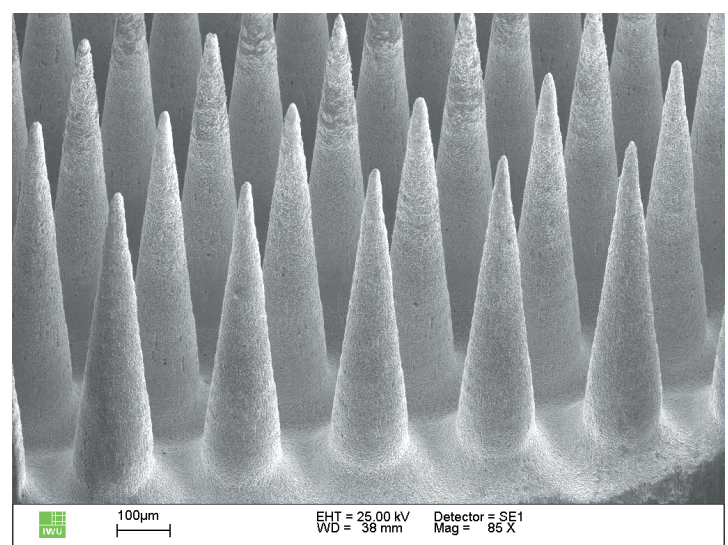

Fig. 9. Array of 121 micro cones (segment view); diameter 150/16 $\mu \mathrm{m}$, height $825 \mu \mathrm{m}$, centre-centre-distance $250 \mu \mathrm{m}$, steel

\subsection{Ultrasonic - Indirect}

The utilisation of the great advantages of direct ultrasonic vibration induced in the tool or workpiece presents the user with the difficulty of designing a suitable actuation- and clamping setup that can withstand the high acceleration forces. Since these are proportional to the mass of the system, exciting a large part is challenging.

One approach to implementing a similar setup without exciting the workpiece or tool is to integrate an additional vibrating part to overlay the discharge area with a high intensity ultrasonic field. 
Possible setups consist of an ultrasonic actuator/ transducer and a tuned sonotrode that amplifies the vibration amplitude to the targeted value. The sonotrode is immersed in the dielectric and arranged in a way that the high intensity near field of the ultrasonic is aligned to the machining zone (Fig. 10).

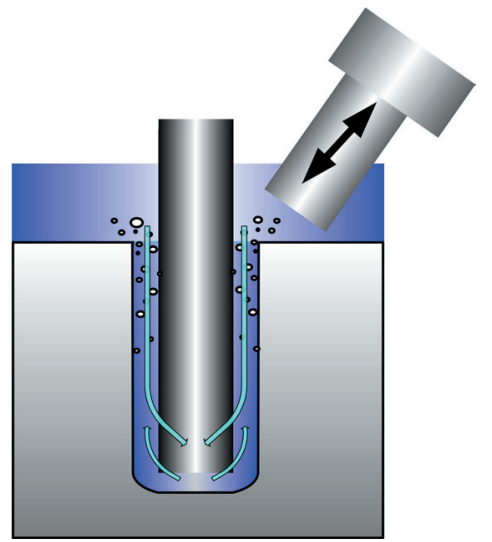

Fig. 10. Indirect ultrasonic superposition in $\mu E D M$

The usage of external equipment allows for an even higher velocity at the sonotrode tip. The experiments conducted are based on sonotrode tip speeds of up to $2.2 \mathrm{~m} / \mathrm{s}$ at frequencies of $24 \mathrm{kHz}$ and amplitudes of $30 \mu \mathrm{m}$ peak-peak which lead to intense movements within the dielectric.

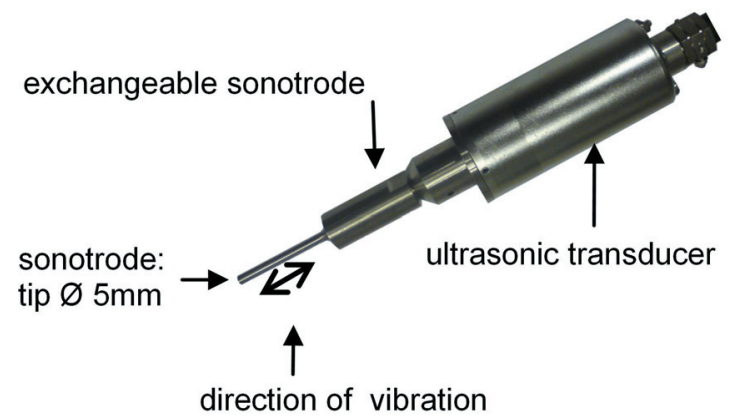

Fig. 11. Equipment for indirect ultrasonic vibration superposition

When applying indirect ultrasonic superposition it has to be considered that, because of the orientation of the sonotrode noncoaxial to the feed direction, the tool electrode is also subject to excitation through the pressure waves within the dielectric. This is especially so in the case of a vibration in the eigenfrequencies of the tool, where electrode resonance movement can adversely affect the process. Consequently, this frequency range should be avoided.

Although in contrast to direct vibration there is no variation in the discharge gap width, the influence on the dielectric can be seen as similar.
Within the near field, one important effect is clearly visible. High intensity, fast movement causes cavitation, meaning the creation and collapse of small gas bubbles through pressure variation (to below the vapour pressure of the liquid) in the medium. In addition, cavitation occurs more easily when the homogeneity of the liquid is disturbed by particles. Within the discharge gap, particles are created during the EDM process and, as a result, cavitation is additionally facilitated. Stuck to the particles, the gas bubbles of the cavitation phenomenon can become stable gas bubbles that rise and create a stream transporting particles out of the discharge gap [4], which contributes to improved flushing and homogenisation.

In practice, a challenge is the alignment and delivery of the high intensity pressure waves to the discharge gap. The angle between the feed direction and the ultrasonic wave front is an important parameter that, for deep structures, should be minimised.

Using the indirect ultrasonic superposition with angles around $60^{\circ}$, a stabilisation of the bore geometry to a more cylindrical shape and a process speed enhancement of $5 \%$ can be achieved.

A further improvement is expected when a coaxially induced ultrasonic vibration is applied, as is currently being investigated. The setup used is shown in Fig. 12.

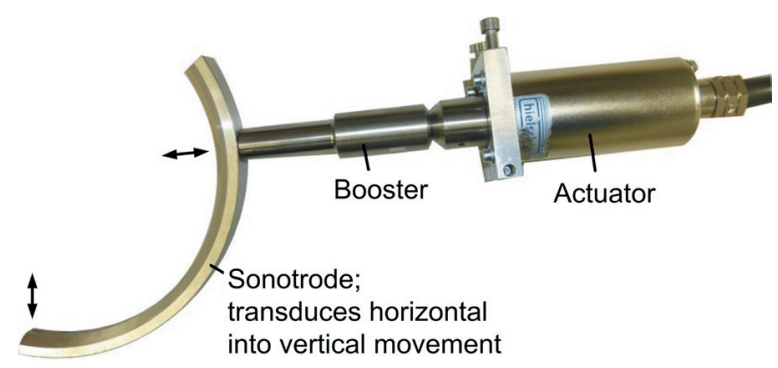

Fig. 12. Setup for coaxial indirect ultrasonic vibration

The conducted experiments, however, highlight another crucial factor in the micro-EDM of very small bores: the length of the electrode tool. The required mechanical stability can, in principle, be delivered by tungsten carbide tools, but this leads to changed boundary conditions for the micro-EDM: primarily, a decrease in electrical conductivity. In our tests using $\varnothing 90$ and $\varnothing 150 \mu \mathrm{m}$ tool electrodes and, due to geometrical restrictions, a free tool length of $>14 \mathrm{~mm}$ - a significant decrease in process speed and stability occurred. Further investigations showed a correlation to the free tool length FTL (distance from clamping/ electrical contact to electrode tip). The process 
changes its characteristics when the FTL exceeds a certain length to diameter ratio. Signal analysis revealed that the maximum peak current decreases substantially with increasing FTL.

A second obstacle is the sensitivity of the tool to orthogonal forces such as adhesion. Experiments show that even when using tungsten carbide tools, from a FTL aspect ratio of more than 50 onwards, bore diameter and machining time increase exponentially. Therefore, adapted machine tool setups have to be designed, allowing for a minimum FTL while still enabling coaxial vibration coupling. One approach is the use of membrane-shaped piezoceramic actuators. The design for such setup is currently taking place.

\section{EDM OF NONCONDUCTING CERAMICS}

\subsection{Demand and Approach}

Ceramic materials are, due to their extraordinary properties such as high hardness and biocompatibility, increasingly used in micro parts. Its machining, however, is difficult, and mostly slow and expensive grinding processes are used [5]. Electro discharge machining with its nearly forceless behaviour and independence of the material's hardness and brittleness seems to be a suitable process, but is mainly limited to conductive materials. In research, several approaches have been taken to machining nonconductive ceramics using EDM, with the 'assisting electrode' method developed by Mohri and Fukuzawa [6] and [7] leading to the successful machining of zirconium dioxide materials.
To achieve machinability with $\mu \mathrm{EDM}$, the process has to be initially started by first delivering an electric circuit through the conductive starting layer as shown in Fig. 13.

While machining the starting layer, the dielectric (hydrocarbon oil) is cracked, providing conductive carbon that settles down onto the ceramic surface. Thus, a new conductive layer is generated that enables the next discharges to take place.

By tightly controlling the process environment, this sequence of removing the layer, including underlying base material, and creating a new thin layer can be stably repeated.

In previous studies, the assisting electrode approach has been successfully adapted to a vibrationassisted $\mu \mathrm{EDM}$ setup of a biocompatible zirconium dioxide compound in order to examine the effects of different frequencies and amplitudes on process speed and achievable geometries [8] and [9].

\subsection{Modifications and Ceramic $\mu$ EDM Process}

A standard $\mu E D M$ machine tool was used for the experiments (Sarix T1-T4) and was modified to comply with the special requirements of $\mu \mathrm{EDM}$ of nonconductive ceramics.

The modifications developed consist of a basin, filled with dielectric oil and equipped with a peristaltic pump for flushing and filtering of the medium independent from the main dielectric circulation, as well as an active workpiece clamping unit that can be excited with a low-frequency vibration of up to $f=$ $1000 \mathrm{~Hz}$ at an amplitude of $20 \mu \mathrm{m}$ peak-peak.
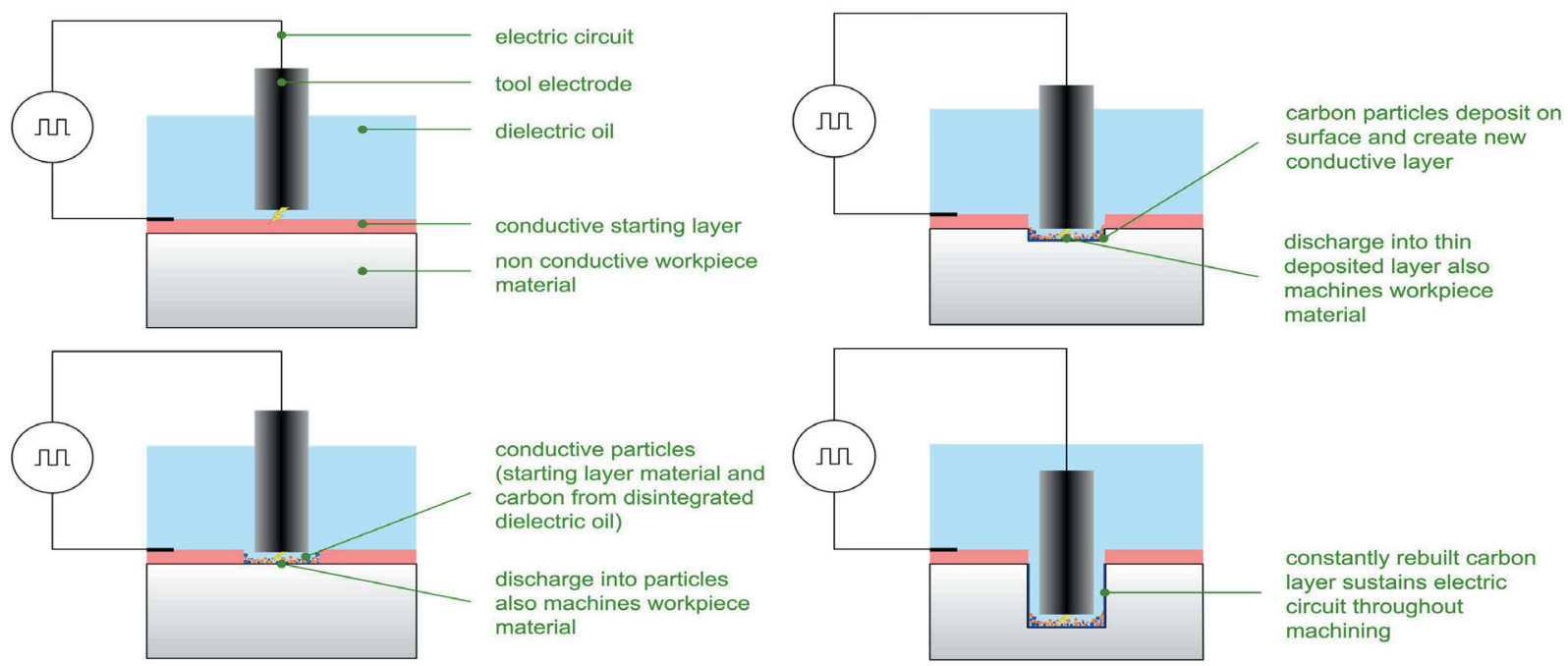

Fig. 13. Assisting electrode scheme for machining nonconductive ceramic materials 
A common silver varnish with $45.9 \%$ silver content is used to create the starting layer. It is applied with a paintbrush. The uniformity of the starting layer is very important, therefore, after drying, it is tested by measuring the resistance using a multimeter.

The thickness of the layer is $20 \mu \mathrm{m}$.

In previous tests, it was found that machining of nonconducting ceramic $\mathrm{ZrO}_{2}$ material only takes place with a cathodic polarity. The generator should be set accordingly.

The electrode wear can be noticeably reduced by introducing tool rotation; however, with values of $\approx 75 \%$ relative wear there is still a high reduction potential.

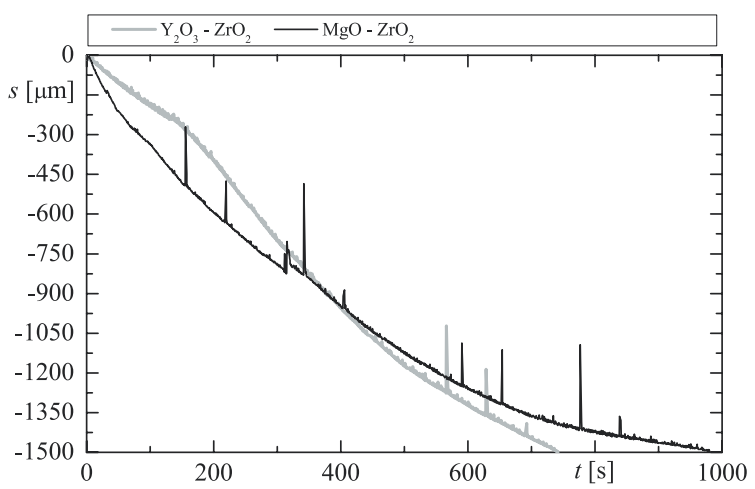

Fig. 14. Tool feed (s) during $\mu E D M$ of nonconducting $\mathrm{ZrO}_{2}$ ceramics (tool $\emptyset 120 \mu \mathrm{m}$ )

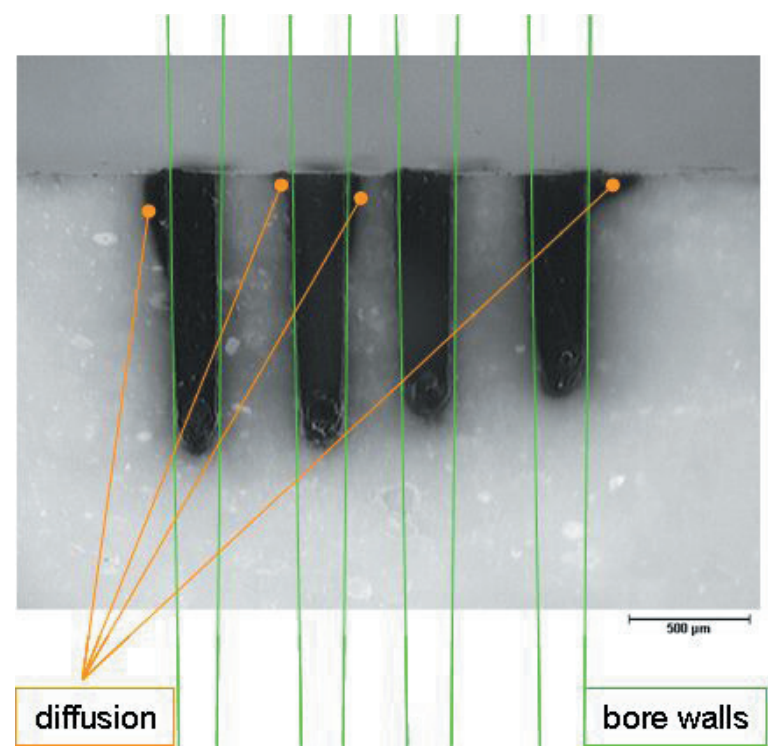

Fig. 15. Cross section of $\mu$ EDM bores in ZrO2 ceramic, diameter $200 \mu \mathrm{m}$, tool $\varnothing 120 \mu \mathrm{m}$

Pulse analysis shows a change in the discharge shape from machining the starting layer, where short pulses similar to discharges in metal approximately
$100 \mathrm{~ns}$ long are observed, to machining the base material. There, the discharge shape changes to a short peak followed by a long hold period at low currents. This type of discharge can last up to $8 \mu$ s and its second part is expected to be the period where the carbon layer is deposited.

\subsection{Machinability of Nonconductive Ceramics}

Using the proposed setup, a successful machining of $\mathrm{ZrO}_{2}$ ceramics is performed, even after the starting layer is completely removed (Fig. 14).

Bores with an aspect ratio 5 could be manufactured using $\mathrm{Y}_{2} \mathrm{O}_{3}$ - (Fig. 15) as well as $\mathrm{MgO}$ stabilised $\mathrm{ZrO}_{2}$ material

\section{CONCLUSIONS}

\subsection{Vibration Superposition}

The $\mu E D M$ process benefits extraordinarily from vibration superposition. Where conventional $\mu \mathrm{EDM}$ processes come to their limits and lose their reproducibility, the process is now stabilised in terms of shape accuracy and machining time.

This is already achievable using indirect vibration, induced via the dielectric. By minimising the angle between the tool feed and wave front direction, further improvements are possible. However, new setups have to be designed to reduce the free tool length to a minimum in order to achieve stability and immunity to external forces, especially when using small tool diameters of less than $100 \mu \mathrm{m}$. Membrane-shaped piezoceramic actuators using bending mode appear to deliver beneficial properties.

With direct application of vibrations, higher aspect ratio structures can be machined and the process speed is improved. Here, the vibration frequency is the crucial point.

Low-frequency vibration mostly reduces the deviation of the process, both in terms of machining time and shape accuracy. A rise in machining speed in the range of approximately $10 \%$ compared to an optimised conventional process is seen.

Using direct ultrasonically aided micro-EDM, the process speed can be raised by up to $40 \%$. The discharge gap state benefits additionally from the cutting off of long arc discharges and geometric deviations are further reduced.

The direct ultrasonic vibration of the tool or workpiece enables the machining of complex structures with very high aspect ratios, as well as 
enabling bores of less than $90 \mu \mathrm{m}$ in diameter with aspect ratios $>40$ for metallic materials.

\subsection{Ceramics}

Using the assisting electrode method and the proposed experimental setup, micro bores can be successfully machined in $\mathrm{MgO}$ - and $\mathrm{Y}_{2} \mathrm{O}_{3}$-stabilised $\mathrm{ZrO}_{2}$ ceramic materials. The use of tool electrode rotation leads to an increase in machining speed and reduces tool wear. While at the beginning of machining the starting layer discharges similar to those in metal$\mu E D M$ can be observed, a transition into so-called ceramic discharges takes place that are characterised by a smaller peak current followed by a long constant low current flow. These make up the majority of discharges when machining the ceramic base material. The process that is used for $\mathrm{Y}_{2} \mathrm{O}_{3}-\mathrm{ZrO}_{2}$ can also be used for $\mathrm{MgO}-\mathrm{ZrO}_{2}$.

\subsection{New Boundaries of $\mu$ EDM}

Micromachining is facing new challenges with the constantly growing demand for smaller, more accurate structures in new, emerging materials. Electro discharge machining has the potential to fulfil those needs in hard to machine materials.

The application of hybrid processes to enhance stability by influencing the flushing and discharge gap state has shown great potential in the machining of conducting materials. Ultrasonic superposition excels at stabilising and thus accelerating the $\mu \mathrm{EDM}$ of very deep, precise structures.

With the integration of the assisting electrode method, materials considered to be very hard to machine, such as engineered and biocompatible ceramics, have become available for new applications that require geometries and aspect ratios that the existing processes cannot deliver.

Combining the two approaches, new possibilities for the design and manufacture of complex, high- accuracy micro parts in high-performance engineering materials can be utilised.

\section{REFERENCES}

[1] Kunieda, M., Lauwers, B., Rajurkar, K. Schumacher, B. (2005). Advancing EDM through Fundamental Insight into the Process. CIRP Annals - Manufacturing Technology, vol. 54, p. 64-87, DOI:10.1016/S00078506(07)60020-1.

[2] Liao, Y.S., Chang, T.Y., Chuang, T.J. (2008). An on-line monitoring system for a micro electrical discharge machining (micro-EDM) process. Journal of Micromechanics and Microengineering, vol. 18, no. 3, 035009, DOI:10.1088/0960-1317/18/3/035009.

[3] Garn, R., Schubert, A. Zeidler, H. (2011). Analysis of the effect of vibrations on the micro-EDM process at the workpiece surface. Precision Engineering, vol. 35, no. 2, p. 364-368, DOI:10.1016/j. precisioneng.2010.09.015.

[4] Yeo, S.H., Tan, L.K. (1999). Effects of ultrasonic vibrations in micro electro-discharge machining of microholes. Journal of Micromechanics and Microengineering, vol. 9, no. 4, p. 345-352, DOI: 10.1088/0960-1317/9/4/310.

[5] Freiman, S. (ed.) (2007). Global Roadmap for Ceramics and Glass Technology, John Wiley \& Sons, Inc., Hoboken.

[6] Mohri, N., Fukuzawa, Y., Tani, T., Saito, N. Furutani, K. (1996). Assisting electrode method for machining insulating ceramics. CIRP Annals - Manufacturing Technology, vol. 45, p. 201-204.

[7] Mohri, N., Chen, S., Fukuzawa, Y., Tani, T., Sata, T. (2002). Some considerations to machining characteristics of insulating ceramics -towards practical use in industry. CIRP Annals - Manufacturing Technology, vol. 51, p. 161-164.

[8] Schubert, A. Zeidler, H. (2009). Manufacturing of Nonconductive $\mathrm{ZrO}_{2}$ Ceramics with MicroEDM. Proceedings of the Euspen 9th International Conference, vol. 2, p. 6-9.

[9] Schubert, A. Zeidler, H. (2010). Implementation of micro-EDM process for machining of nonconductive ceramics. Proceedings of the $16^{\text {th }}$ International Symposium on Electromachining ISEM-XVI, p. 565570. 